\title{
Can the Nociception Coma Scale-Revised Be Used in Patients With a Tracheostomy?
}

\author{
Nicolas Lejeune, MD, ${ }^{a, b, c}$ Aurore Thibaut, $\mathrm{PhD},{ }^{a}$ Géraldine Martens, MSc, ${ }^{a}$ \\ Charlotte Martial, $\mathrm{PhD}$, ${ }^{a}$ Sarah Wannez, PhD, ${ }^{a}$ Steven Laureys, MD, PhD, \\ Camille Chatelle, PhD ${ }^{\mathrm{a}, \mathrm{d}}$
}

From the ${ }^{a}$ GIGA-Consciousness, Coma Science Group, University of Liegge, Belgium; ${ }^{b}$ Institute of NeuroScience, Université catholique de Louvain, Brussels, Belgium; 'Centre Hospitalier Neurologique William Lennox, Groupe Hospitalier Saint-Luc, UCL, Ottignies, Belgium; and ${ }^{d}$ Laboratory for NeuroImaging of Coma and Consciousness, Department of Neurology, Massachusetts General Hospital, Harvard Medical School, Boston, Massachusetts.

\begin{abstract}
Objective: To investigate the influence of the presence of a tracheostomy tube to assess pain with the Nociception Coma Scale-Revised (NCS-R) in patients with disorders of consciousness (DOC).

Design: A cohort study in which patients were evaluated at a single time point.

Setting: Patients were evaluated in a tertiary care hospital.

Participants: Patients $(\mathrm{N}=125)$ (unresponsive wakefulness syndrome [UWS]: 46 patients, minimally conscious state [MCS]: 74 patients, emerging from MCS [eMCS]: 5 patients, mean age: $46 \pm 16 \mathrm{y}$, time since injury: $817 \pm 1280 \mathrm{~d}$ ) in a convenience sample were evaluated with the NCS-R after noxious stimulation.

Interventions: Not applicable.

Main Outcome Measures: We compared the NCS-R scores of patients with and without tracheostomy with a Mann-Whitney $U$ test. A secondary outcome was to evaluate the influence of the presence of a tracheostomy on the previously described cutoff score of 2 .

Results: The presence of a tracheostomy was associated with lower verbal subscores $(P=.002)$ as well as total scores $(P=.039)$. The cutoff score of 2 remained valid for the group of patients with tracheostomy with a high sensitivity $(71.43 \%)$ and specificity $(89.29 \%)$, as well as when we excluded the verbal subscore of the NCS-R (sensitivity $=83.2 \%$ and specificity $=92.4 \%$ ).

Conclusion: Our study confirms the validity of the NCS-R in DOC patients with a tracheostomy. However, the presence of a nonspeaking tracheostomy should be clearly mentioned when applying the NCS-R, because it significantly lowers the verbal subscore.

Archives of Physical Medicine and Rehabilitation 2020;101:1064-7

(C) 2019 by the American Congress of Rehabilitation Medicine

After a severe brain injury leading to coma, some patients will remain in a pathological state of impaired consciousness often referred to as disorders of consciousness (DOC), encompassing

Supported by the University and University Hospital of Liege, the Belgian National Funds for Scientific Research (FRS-FNRS), the European Union's Horizon 2020 Framework Programme for Research and Innovation under the specific Grant No. 785907 (Human Brain Project SGA2), the Luminous project (EU-H2020-fetopenga686764), the Center-TBI project (FP7-HEALTH-602150), the Public Utility Foundation Université Européenne du Travail, Fondazione Europea di Ricerca Biomedica, the Bial Foundation, the European Space Agency, the Mind Science Foundation, and the European Commission. N.L. is a PhD fellow, A.T. is a postdoctoral fellow, and S.L. is research director at FRS-FNRS. C.C. is a Marie Sklodowska-Curie fellow (H2020-MSCA-IF-2016-ADOC752686).

Disclosures: none.

(1) patients showing only reflexive behaviors (unresponsive wakefulness syndrome [UWS], ${ }^{1}$ also known as vegetative state) and (2) patients exhibiting fluctuating purposeful behaviors, but unable to functionally communicate (minimally conscious state $[\mathrm{MCS}]) .^{2}$ Seminal studies showed that DOC patients may present at least a partial cortical process of noxious stimuli. More specifically, patients in MCS, at a group level, showed the same pattern of functional activation as healthy participants. ${ }^{3,4}$ These findings have had a significant clinical and ethical effect and highlight the necessity to develop bedside tools to identify and manage pain in this population. In 2010, a behavioral scale was developed specifically for patients with DOC, the Nociception
\end{abstract}


Coma Scale-Revised (NCS-R). 5,6 This scale encompasses 3 subscales, evaluating motor, verbal, and facial expressions, each one ranging from 0 to 3 , with a maximal total score of 9 (box 1). In a recent multicenter study, a cutoff score of 2 was identified $^{7}$ to detect responses related to nociceptive stimuli.

If it has been shown useful to manage pain in intensive care patients, ${ }^{8-10}$ the frequent presence of a tracheostomy tube may prevent patients to emit any sound, secondary to the endotracheal tube by-passing the vocal chords, resulting in a low to null score on the verbal subscale. In this retrospective study, we investigated the influence of the presence of a tracheostomy on the verbal subscale and on the NCS-R total score. We also aimed to replicate the previously defined cutoff of 2 and to investigate if it would be different in a subgroup of patients with a tracheostomy.

\section{Methods}

\section{Participants}

Participants were recruited from the intensive care unit and neurology ward of the University Hospital of Liège, from neurorehabilitation centers and nursing homes that are part of the Belgian federal network for DOC patients, between 2011 and 2017. Inclusion criteria were (1) age $>16$ years; (2) no administration of neuromuscular blockers or sedation within the 24 hours of enrollment; (3) the presence of periodic eye opening; (4) a diagnosis of UWS, MCS, or emergence from MCS (eMCS) without functional communication, based on behavioral assessment performed using the Coma Recovery Scale-Revised. ${ }^{11}$ Exclusion criteria were (1) past medical history of brain injury; (2) developmental, psychiatric, or neurologic illness resulting in documented functional disability up to time of the injury; and (3) upper limb contusions, fractures, or flaccid paralysis. A subset of patients $(n=64)$ were previously included in a study published elsewhere. ${ }^{6}$ The study was approved by the Ethics committee of the Faculty of Medicine of the University of Liège, and the patient's legal representatives gave their written informed consent.

Two samples of patients assessed with the NCS-R were included for analyses. The first one included patients for whom we had information on the presence or absence of tracheostomy. For the secondary analyses, we included any patient who underwent NCS-R assessments.

\section{Procedure}

The NCS-R was administered in 2 different conditions: (1) baseline and (2) noxious condition. During baseline, we observed the patient's spontaneous behaviors for 60 seconds. ${ }^{11}$ During the noxious condition, we applied pressure on the nailbed of the middle finger of the right and left hand ${ }^{11,12}$ for a minimum of 5 seconds and stopped as soon as a behavioral response was observed. Behavioral responses were recorded 10 seconds after each stimulation. ${ }^{11}$ The highest score obtained across right and left sides stimulation was considered. To ensure a sufficient level

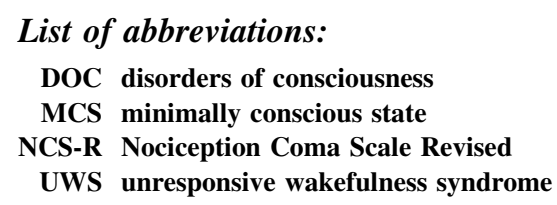

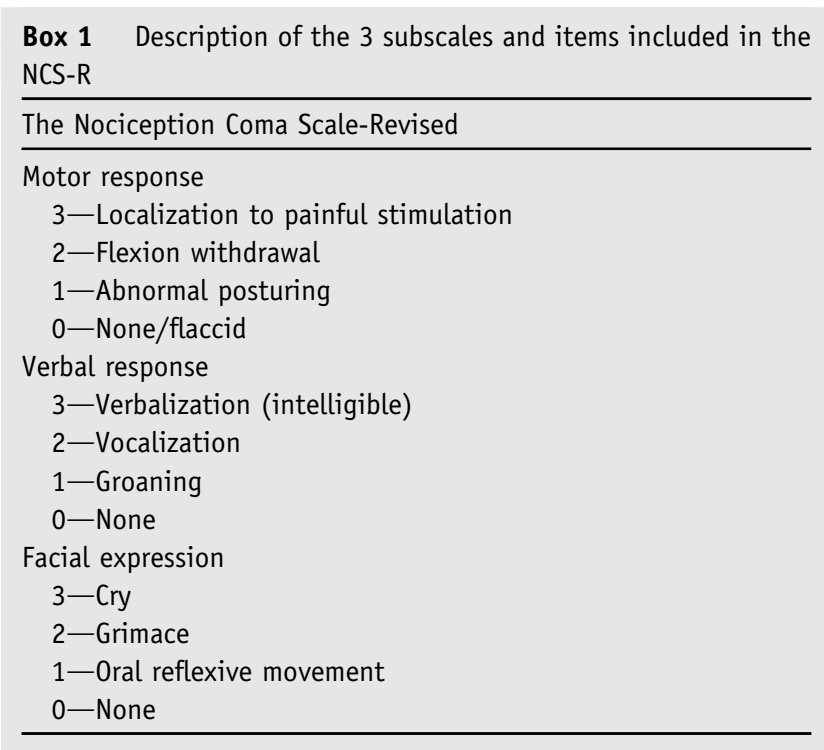

of arousal, each condition was administered while patients showed spontaneous eye opening. The entire procedure lasted $<5$ minutes.

Patients' level of consciousness was assessed before or after this procedure with the Coma Recovery Scale-Revised. ${ }^{13}$

\section{Statistics}

Statistical analyses were performed using GraphPad Prism 7. ${ }^{\text {a }}$

In the first step, Mann-Whitney $U$ tests were used to investigate the difference in NCS-R subscores and total scores in response to noxious stimulation between patients with and without a tracheostomy tube. We set significance at $P<.05$ (one-tailed).

In a second step, receiver operating characteristic analyses were used to determine the best cutoff score allowing to differentiate the noxious condition versus baseline using the NCS-R total scores and the sum of both the motor and facial expression subscales excluding the verbal one. We ran those analyses on a larger sample of patients assessed with the NCS-R as well as on a subset of patients with tracheostomy.

\section{Results}

For the first step, 65 patients (UWS [ $n=25]$, MCS [ $n=35]$, eMCS $[\mathrm{n}=5])$ were included, of which 28 had a tracheostomy $(43 \%)$ (UWS [61\%], MCS [32\%], eMCS [7\%]). Mean age of the population was $42 \pm 13$ years old (time since injury $1198 \pm 1495$ d). Lower scores were observed on the NCS-R verbal subscores $(P=.002)$ and total scores $(P=.039)$ in the tracheostomy group as compared with patients without a tracheostomy in response to noxious stimulation (fig 1). Moreover, there was no difference in facial $(P=.241)$ and motor subscores $(P=.967)$ between both groups.

For the second step, we included 125 DOC patients (UWS $[n=46]$, MCS [ $n=74]$, eMCS $[n=5]$ patients, mean age: $46 \pm 16$ year old, time since injury: $817 \pm 1280 \mathrm{~d}$ ) assessed with the NCS-R. The receiver operating characteristic analyses reported a score of $\geq 2$ as the best threshold for nociception with a sensitivity of $87.2 \%$ and a specificity of $79.2 \%$ for differentiating noxious condition from baseline (fig 2). Removing the verbal subscale 


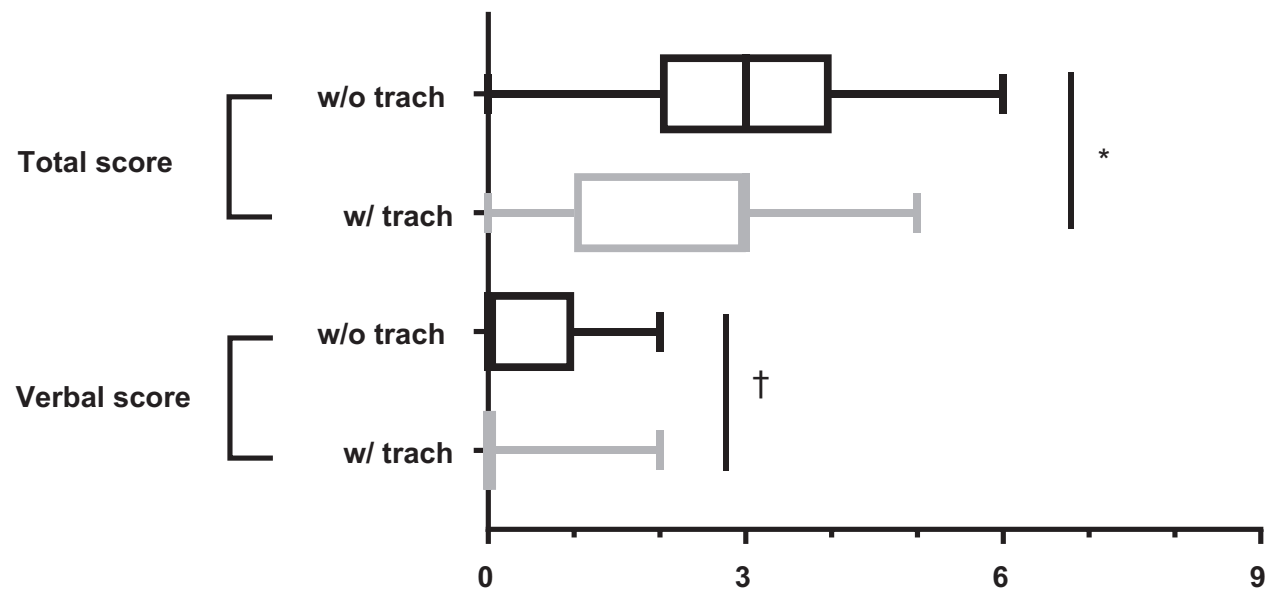

Fig 1 Median (and interquartile range) of NCS-R total scores (upper rows) and verbal subscores (lower rows) in response to noxious stimuli in subgroups of patients without $(\mathrm{n}=37)$ (on the graph $w / 0$ trach) or with a tracheostomy $(\mathrm{n}=28)$ (on the graph $w /$ trach). Asterisks mark significant difference between the scores of the 2 groups ( ${ }^{*}$ one-tailed $P<.05$ for total scores and ${ }^{\dagger}$ one-tailed $P=.01$ for verbal subscores).

(motor+facial score) did not modify the cutoff, with a score of 2 associated with a sensitivity of $83.2 \%$ and a specificity of $92.4 \%$ (see fig 2).

The same analyses performed on a sample of 28 patients with tracheostomy led to the same cutoff score of 2 at the NCS-R total score, with a lower sensitivity (71.43\%) and specificity $(89.29 \%)$, while removing the verbal subscale led to the same results (see fig 2) (see also supplemental table S1, available online only at http://www.archives-pmr.org/, for more details).

\section{Discussion}

We here showed that the presence of a tracheostomy is associated with a lower score on the verbal subscale, resulting also in a lower total score. Patients with tracheostomy also tended to display similar pattern of responses at the motor and facial subscales of the NCS-R when compared to nontracheostomized patients. These findings are in line with our hypothesis that the NCS-R scores are mainly influenced by the presence of a tracheostomy itself, and the difference is not due to a difference in the type of DOC or disease severity.

We also replicated the previously reported cutoff score of $2^{7}$ to identify nociception, which remained the same after removal of the verbal subscore or when focusing on a subgroup of patients with tracheostomy.

\section{Study limitations}

Future studies should use a Newton meter to control stimulus intensity and limit interrater variability on the amount of pressure administered during noxious condition. Investigating NCS-R responses in patients with a speaking tracheostomy versus

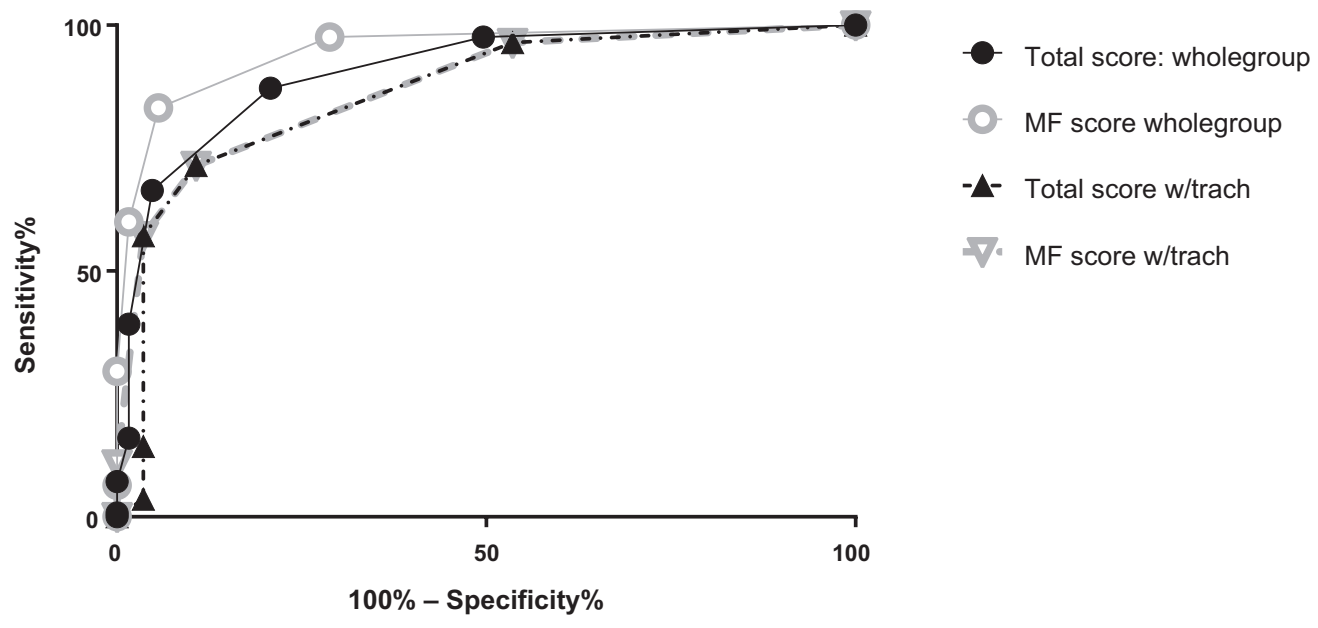

Fig 2 The receiver operating characteristic curves representing the relation between sensitivity and specificity for the different potential threshold scores allowing to discriminate a NCS-R score at baseline (ie, resting scores) and a NCS-R score after noxious stimulations using different scores (ie, NCS-R total scores or total score without the verbal subscore [motor+facial]) or population (ie, for the whole group) $(N=125)$ and for the subgroup of patients with tracheostomy $(n=28)$. Area under curve values as well as values of each point of the curves are collected in supplemental table S1. MF, motor+facial. 
nonspeaking tracheostomy would also enable to confirm the hypothesis that a difference is solely due to the medical device itself. Finally, the clinical implication of the cutoff score of 2 should also be further investigated, because the score of 2 can easily be reached with only reflexive behaviors (ie, flexion withdrawal), limiting its interest in a clinical setting to identify a (potentially) painful condition.

\section{Conclusion}

Our study confirms the validity of the NCS-R in DOC patients with a tracheostomy. However, the presence of a nonspeaking tracheostomy should be clearly mentioned when applying the NCS-R because it significantly lowers the verbal subscore and total score.

\section{Supplier}

a. GraphPad Prism 7; GraphPad Software.

\section{Keywords}

Brain injuries; Minimally conscious state; Nociception; Pain; Rehabilitation; Tracheostomy

\section{Corresponding author}

Nicolas Lejeune, MD, GIGA-Consciousness, Coma Science Group, University of Liège, CHU Sart Tilman, GIGA-Research B34-Quartier Hôpital, 11, Avenue de l'Hôpital, 4000 Liège, Belgium. E-mail address: nicolas.lejeune@uclouvain.be.

\section{Acknowledgments}

We thank the whole staff from the Neurology, Radiodiagnostic, and Nuclear Medicine departments, University Hospital of Liege and the patients. We are highly grateful to the members of the
Liège Coma Science Group for their assistance in clinical evaluations.

\section{References}

1. Laureys S, Celesia GG, Cohadon F, et al. Unresponsive wakefulness syndrome: a new name for the vegetative state or apallic syndrome. BMC Med 2010;8:68.

2. Giacino JT, Ashwal S, Childs N, et al. The minimally conscious state: definition and diagnostic criteria. Neurology 2002;58:349-53.

3. Laureys S, Faymonville ME, Peigneux P, et al. Cortical processing of noxious somatosensory stimuli in the persistent vegetative state. Neuroimage 2002;17:732-41.

4. Boly M, Faymonville ME, Schnakers C, et al. Perception of pain in the minimally conscious state with PET activation: an observational study. Lancet Neurol 2008;7:1013-20.

5. Schnakers C, Chatelle C, Vanhaudenhuyse A, et al. The Nociception Coma Scale: a new tool to assess nociception in disorders of consciousness. Pain 2010;148:215-9.

6. Chatelle C, Majerus S, Whyte J, et al. A sensitive scale to assess nociceptive pain in patients with disorders of consciousness. J Neurol Neurosurg Psychiatry 2012;83:1233-7.

7. Chatelle C, Hauger SL, Martial C, et al. Assessment of nociception and pain in participants in an unresponsive or minimally conscious state after acquired brain injury: the relation between the Coma Recovery Scale-Revised and the Nociception Coma Scale-Revised. Arch Phys Med Rehabil 2018;99:1755-62.

8. Chatelle C, De Val MD, Catano A, et al. Is the Nociception Coma Scale-Revised a useful clinical tool for managing pain in patients with disorders of consciousness? Clin J Pain 2016;32:321-6.

9. Vink P, Eskes AM, Lindeboom R, et al. Nurses assessing pain with the Nociception Coma Scale: interrater reliability and validity. Pain Manag Nurs 2014;15:881-7.

10. Suraseranivongse S, Yuvapoositanont P, Srisakkrapikoop P, et al. A comparison of pain scales in patients with disorders of consciousness following craniotomy. J Med Assoc Thai 2015;98:684-92.

11. Giacino J, Kalmar K, Whyte J. The JFK Coma Recovery ScaleRevised: measurement characteristics and diagnostic utility. Arch Phys Med Rehabil 2004;85:2020e9.

12. Teasdale G, Jennett B. Assessment of coma and impaired consciousness: a practical scale. Lancet 1974;2:81e4.

13. Kalmar K, Giacino JT. The JFK Coma Recovery Scale-Revised. Neuropsychol Rehabil 2005;15:454-60. 